\title{
Enzo Caffarelli
}

Università di Roma "Tor Vergata"

\section{Replicazioni toponimiche nello spazio geografico e nello spazio linguistico}

I toponimi, qualunque siano la natura, la storia, le dimensioni insediative del referente, spesso non esauriscono il loro percorso fermandosi a designare un'entità unica, ma viaggiano nel tempo e nello spazio; e tale spazio può avere una duplice natura: geografica e linguistica.

Il nome di luogo viaggia nello spazio geografico attraverso i processi di duplicazione e, non raramente, di replicazione multipla, che hanno alla base numerose motivazioni, legate alle migrazioni dei popoli, alle apparenze geomorfologiche o ambientali o di antropizzazione, alle commemorazioni e tributi, a ragioni economiche e turistiche. Viaggia invece nello spazio linguistico attraverso due potenzialità del nome proprio che si traducono in processi tipici e sovente collegati tra loro: la lessicalizzazione (dal nome proprio al nome comune) con l'eventuale successiva rionimizzazione (dal nome comune al nome proprio) e la transonimizzazione (dal nome proprio a un altro nome proprio appartenente a una tipologia onimica differente).

In queste pagine saranno portati alcuni esempi di macro- e microtoponimi italiani (tenendo presente, ma non tornando sulla discussione in merito, che la distinzione tra queste due categorie è sottile e perlopiù opinabile ${ }^{1}$ che hanno percorso un cammino nello spazio geografico e / o in quello linguistico sia in Italia e nell'italiano, sia, soprattutto, in altre lingue, nazioni e continenti.

Esistono località italiane $\mathrm{i}$ cui nomi sono noti nel mondo per le bellezze artistiche, la cultura, i prodotti tipici artigianali e alimentari; altre sono divenuti note o addirittura celeberrime senza un merito diretto: sono casi in cui il toponimo originale ha assunto un impredicibile significato e per le più svariate ragioni è oggi usato come nome proprio o nome comune in molte lingue. Il fenomeno riguarda qualsiasi tipologia di nome proprio; in àmbito antroponimico e crematonimico, si pensi alla voce paparazzo: in origine soprannome per indicare un papero con suffiso spregiativo (se non un nome di mestiere per 'prete sarto'), poi fissatosi in

\footnotetext{
${ }^{1}$ Cfr. Caffarelli 2015a; 2015b; Poccetti 2014.
} 
un nome di famiglia, tipico del centro della Calabria, quindi scelto come soprannome o cognome per un personaggio finzionale (il fotoreporter mondano del film di Federico Fellini La dolce vita, 1960), di lì in poi divenuto nome comune per indicare una particolare categoria di fotografi legati ai divi dello spettacolo, e poi rionimizzato in centinaia di insegne di ristoranti, pizzerie, hotel, saloni di bellezza, boutique di gioielli, ecc. [Caffarelli 2015b].

Ma, limitando l'analisi ai toponimi, possiamo avviare l'indagine con alcuni esiti del fenomeno migratorio che dall'Italia ha condotto negli altri continenti, in particolare nell'Europa centro-settentrionale, nelle due Americhe e in Australia, circa 28 milioni di cittadini tra gli ultimi decenni del XIX secolo e gli anni 70 del XX e che conta oggi su 80 milioni di italiani o di oriundi residenti fuori d'Italia.

\section{Replicazioni nello spazio geografico}

Se consideriamo, per esempio, i toponimi della Campania replicati negli Stati Uniti d'America, scopriamo che i centri abitati di nome Sorrento sono almeno 8, oltre a un tunnel nell'Ohio, una baia nel Maine, ecc.; Pompeii (con due $i$ ) si trova nel Michigan (e un corso d'acqua omonimo nel Kentucky); un parco Benevento è nel Massachussetts; Capri dà nome a villaggi, miniere, laghi e naturalmente isole; Naples è nome di luogo diffusissimo, soprattutto in California e in Florida, e almeno presso New York un paese si chiama proprio Napoli. Numerosi i nesonimi replicati: la città di Ponza si trova nella contea Bell, nello stato del Kentucky; oltre 20 sono i toponimi con il nome Capri: isole anche in Alabama, Florida e South Carolina, laghi nel Montana e nello Stato di New York, eliporto in Colorado e... un paio di grandi shopping center in California. Sicilia e Sardegna figurano con i corrispondenti inglesi: Sicily è un'isola della Florida, della Lousiana e della Pennsylvania, una miniera in California, un paese dell'Illinois; Sardinia è una cittadina nell'Indiana, nell'Ohio, nel South Carolina, presso New York, ecc. Si tratta, qui, perlopiù di riferimenti diretti.

Merita particolare attenzione il caso di Osasco in Brasile, Paese altrettanto ricco di nomi di comuni italiani, specie settentrionali, considerata la provenienza di gran parte degli emigrati (Nova Bassano, Nova Bréscia, Nova Pádua, Nova Trento, Nova Veneza, Nova Verona, Nova Beluno, Val Trentina...). Si tratta in genere di paesi piccoli, ma nello Stato di Saõ Paulo sorgono la città e il municipio di Osasco, prosecuzione dell'area metropolitana di San Paolo; venne fondata negli anni 80 del XIX secolo da Antonio Giuseppe Agu, nativo di Osasco in provincia di Torino, paesino medievale che contava 944 abitanti all'ultimo censimento Istat (2011); l'Osasco brasiliana ne ha invece quasi 750 mila, quinta città dello stato dopo San Paolo, Guarulhos, Campinas e São Bernardo do Campo [DEMIM: 583]. 
Un secondo caso di derivazione toponimica, stavolta indiretta perché mediata da un antroponimo (si tratta dunque di una transonimia bidirezionale), assai poco conosciuto dagli stessi italiani, conduce a Brazzaville, capitale della Repubblica del Congo ${ }^{2}$; ebbene, quel toponimo deriva da Pietro Paolo Savorgnan di Brazzà (1852-1905), il nobile francese d'adozione ma friulano d'origine (sebbene nato a Castel Gandolfo presso Roma) che esplorò per conto del governo di Parigi una vasta area dell'Africa centrale e ottenne, in cambio di protezione, che i francesi governassero la nuova colonia. Savorgnan fondò appunto Brazzaville nel 1880, sul fiume Congo, prima capitale dell'Africa Equatoriale Francese. L'esploratore veniva da uno dei rami della famiglia: i Brazzà o Brazzacco traevano il loro predicato nobiliare da Brazzacco, località nel comune udinese di Moruzzo (dove si trova tuttora una Villa Savorgnan di Brazzà) ${ }^{3}$.

Un terzo caso, qui con una mediazione agionimica, concerne Copertino. Il comune leccese corrisponde al centro californiano di Cupertino, nella contea di Santa Clara in California, cuore abitato della cosiddetta Silicon Valley, una delle capitali mondiali dell'industria informatica e telematica ${ }^{4}$. Cupertino prende il nome nel 1898 da un'azienda vinicola che a sua volta lo aveva tratto da un vicino corso d'acqua, così battezzato nel '700 dal cartografo Petrus Font, cronista dell'esploratore spagnolo Don Juan Bautista de Anza. La scelta era stata compiuta in onore del suo santo patrono in Italia, San Giuseppe da Copertino, il santo "volante" canonizzato nel 1767. Dal nome di un santo, dunque, a un idronimo e di qui a un nome aziendale e infine a un toponimo vero e proprio.

\subsection{La replicazione nell'astrotoponimia}

La replicazione nello spazio geografico interessa anche l'astrotoponimia, ossia l'insieme dei nomi attribuiti a punti specifici della superfici dei pianeti e dei satelliti del sistema solare o alle loro atmosfere [Caffarelli 2004]. Per gli italiani, e per i piemontesi in particolare, Locana, poco più di 2 mila abitanti, a $60 \mathrm{~km}$ da Torino, dialetto franco-provenzale, è la sede della comunità montana Valle Orco e Soana, nell'alto Canavese, nota in passato come la città dei calderai (e la sua frazione Fornolosa per gli spazzacamini); ma per la comunità scientifica interna-

${ }^{2}$ Già Congo francese, Repubblica Popolare del Congo, a lungo internazionalmente chiamata anche Congo-Brazzaville, per distinguerla dall'altro Congo, ex Belga, poi Zaire, ora Repubblica Democratica del Congo (Congo-Kinshasa).

3 Savorgnan di Brazzà fu uno dei pochi colonizzatori di idee aperte, contrario allo schiavismo, allo sfruttamento coloniale e alla violenza. Per questo visse forti contrasti col governo francese, lasciò la Francia per l'Algeria, morì a Dakar (forse avvelenato) ed è tuttora conosciuto e ammirato in Africa più che in Europa. Sepolto ad Algeri, per lui fu costruito un mausoleo, dove ora ne riposano le spoglie, proprio a Brazzaville, che gli ha dedicato anche l'università, un liceo e il viale principale.

${ }^{4}$ Vi risiedono la Apple, la Hewlett Packard, la Symantec e altre notissime aziende. 
zionale, Locana è un piccolo cratere su Marte. I crateri del pianeta rosso, quando non portano nomi di scienziati o di scrittori che hanno contribuito alla sua conoscenza, sono infatti intitolati a città di tutto il mondo che abbiano meno di 100 mila abitanti, e l'Italia è rappresentata 8 volte; con toponimi ancora piemontesi - Neive (Cuneo) e Novara ${ }^{5}$ - e con il Meridione: Mistretta in Sicilia, Nardò in Puglia, Sarno in Campania come inoltre Ercolano, segnalato latinamente come Herculaneaum. Altri toponimi italiani sono finiti su pianeti e satelliti del nostro sistema: Verona è su Miranda, satellite di Urano; qui l'àmbito semantico è dato dai personaggi e dai luoghi letterari di Shakespeare (Miranda è appunto protagonista della Tempesta): la città scaligera denomina una rupe in quanto sede della vicenda di Romeo e Giulietta; lo stesso vale per Mantua, in quanto toponimo dei Two gentlemen from Verona; Syracusa (da La commedia degli errori) e Sicilia, dove s'ambienta Racconto d'inverno.

Su Gaspra, uno degli asteroidi compresi tra Marte e Giove, i crateri sono stati battezzati con nomi di cave e grotte famose; l'Italia è ricordata con Azzurra, la grotta dell'isola di Capri; Stiffe, le cave presso San Demetrio ne' Vestini in Abruzzo; e Castellana, per le grotte nella bassa Murgia barese. Numerosi sono i Comuni italiani ai quali è dedicato un asteroide o pianeta minore. Uno di questi corrisponde a Belgirate comune piemontese del Verbano-Cusio-Ossola; 1'etimologia conduce all'aggettivo etnico bulgarus (DTI: s.v.) ma l'esito moderno del toponimo fa pensare a un "bel" movimento circolare di un asteroide 6 .

\subsection{Replicazione pluritipologica}

Si è detto di Verona e di Mantova nel sistema solare. Ebbene, il nome della città veneta coincide con almeno 18 centri abitati degli Stati Uniti e uno in Canada (ma solo alcuni di questi toponimi derivano direttamente da Verona). Sicuramente connesse alla città italiana sono un piccolo centro californiano, una cittadina nel Maine, una nel Missouri, così come quella dello Stato di New York ${ }^{7}$. Verona è inoltre legata a un medicinale ipnotico, un tempo largamente diffuso, il Veronal; creato da Hermann Fisher per la Bayer nel 1903, anche conosciuto come Barbital,

${ }^{5}$ Comune che peraltro conta più di 100 mila abitanti, cfr. il censimento nazionale Istat del 2001.

${ }^{6}$ Altri asteroidi dedicati a comuni italiani (salvo casi in cui potrebbe trattarsi di un cognome) sono: Assisi, Bassano, Caprara e Caprera, Cremona, Gorizia, La Spezia, Lucca, Messina, Monsummano, Montefiore, Montelupo, Palazzolo, Palermiti e Palermo, Palestrina, Pejo, Pino Torinese, Piteglio, Pordenone, Putignano, Roma. Un altro gruppo di asteroidi porta denominazioni antiche di città italiane, come Bononia, Brixia, Parthenope e Tergest (ossia Bologna, Brescia, Napoli e Trieste) oltre a Venetia; altri ancora corrispondono a coronimi o idronimi: Ciociaria, Liguria, Sicilia, Susa, Verbano, Isonzo, Riparia.

7 Al contrario, il nome del villaggio dello stato del Nebraska deriva dal cognome di una famiglia di pionieri (la famiglia Veronica), che colonizzarono quel territorio; e quello della cittadina della Pennsylvania ha avuto origine dalla fusione di due altri precedenti toponimi, Verner e Iona. 
pare tragga la propria origine dal fatto che il chimico tedesco si trovasse nella città veneta nell'anno della scoperta del medicinale, e che il farmaconimo fosse conseguenza della suggestione prodotta dalla tragedia shakespeariana e dalla città che ne faceva da scenario [La Stella 1990: 208].

Analogamente, Mantua, forma latineggiante per Mantova, è anche una cittadina di Mantua nella provincia di Pinar di Río, nell'isola di Cuba, secondo la leggenda fondata da Antonello Fiorenzano, un avventuriero mantovano al soldo dei francesi, approdato nell'attuale porto di Los Arroyos nei primi del Settecento; si ipotizza però che la città non derivò direttamente il nome dal gruppo dei primi coloni, ma dal nome della nave su cui viaggiavano, il brigantino Mantua (DEMIM: 460-461). Ipotesi non del tutto certa, se si considera anche che il toponimo Mantua, non relazionabile con la città di Mantova, si ritrova replicato almeno in dieci centri degli Stati Uniti e tre in Brasile.

Gli esempi appena citati rappresentano casi di duplicazione pluritipologica, se non vogliamo assimilare un insediamento umano nelle Americhe ai punti di riferimento sulla superficie di un satellite né agli odonimi urbani che pure sono intitolati a Verona a Mantova ${ }^{8}$, così come a migliaia di altri toponimi. Ma un ben più ampio numero di tipologie incontreremo a proposito delle replicazioni negli spazi linguistici.

\subsection{I toponimi teatro di battaglia e i luoghi ameni}

Altri casi tipici di replicazione toponimica si devono a località che sono stati teatri di battaglie [Caffarelli 2014]. Il nome del comune calabrese di Maida (Catanzaro) è assai più conosciuto in Inghilterra che in Italia. Un grande quartiere londinese si chiama infatti Maida Vale con la collinetta Maida Hill e tale è il nome della stazione metropolitana ed è ben noto quale importanza rivestano, nella toponimia urbana, le linee sotterranee determinando una sorta di iperonimia odonimica [Caffarelli 2015a]. Il 4 luglio 1806 il generale John Stuart, alla guida delle truppe inglesi alleate dei Borbone, sconfisse i francesi con un'abile strategia e al ritorno in patria, Londra volle dedicare un'area poubblica a quella battaglia. Un secolo e mezzo dopo, Maida Vale è entrata prepotentemente anche nella storia del cinema, grazie al film Il delitto perfetto di Alfred Hitchcock ${ }^{9}$.

${ }^{8}$ Secondo la banca dati dai SEAT Pagine Gialle Italia, al 2014 risultavano in Italia 270 odonimi Verona e 215 odonimi Mantova.

${ }^{9}$ Nella pellicola del 1954 protagonista è il telefono: attraverso una telefonata Tony Wendice, interpretato da Ray Milland, deve dare il via all'assassinio della moglie Margot (Grace Kelly) affidato a un sicario; per telefonare in casa l'uomo digita l'indicativo che, in Inghilterra, era alfanumerico: sigla della zona + numero; ebbene, la casa dei Wendice si trova a Maida Vale e Tony digita una "M", su cui Hitchcock gioca per intitolare il film, nell'originale, "Dial M for Murder", ossia 'digita M per omicidio'. 
Rivoli, nome-simbolo per parchi, giardini, hotel, ristoranti, teatri, cinema, laghetti artificiali e luoghi ameni in generale in decine di nazioni e continenti, ha intrapreso un percorso onimico legato alla vittoria napoleonica sull'esercito austriaco del gennaio 1797, nell'area di Rivoli Veronese (in Veneto) e al desiderio di ricordare la località gardesana, al ritorno in Francia, intitolandole uno dei più rinomati e illustri tratti di Lungosenna parigini, l'attuale Rue de Rivoli. I giardini, l'atmosfera festosa e l'imponenza della costruzione di tale area di circolazione hanno trasformato Rivoli, via via sottratto a qualsiasi legame trasparente con il luogo della battaglia, in un nome-garanzia internazionale ${ }^{10}$.

Sostenuto probabilmente anche dall'analogia formale, un altro toponimo italiano è divenuto emblema di parchi, ville, giardini, giochi, spettacoli, ipermercati e amenità internazionale; si tratta di Tivoli, antica città del Lazio [Caffarelli 2015a: 100-101]. Nel medesimo àmbito, a Rivoli e Tivoli si aggiunge il nome di uno dei cosiddetti castelli romani, Frascati. Dopo avervi soggiornato, re Gustavo III di Svezia fece costruire a Stoccolma dall'architetto Louis Jean Desprez nel 1791-1792 una villa che chiamò Frescati, con voluta rietimologizzazione (da fresco) per il clima mite che vi aveva incontrato anche d'estate. Nel XIX secolo il nome si è esteso fino a denominare un'ampia area verde della capitale svedese, dove hanno trovato sede la Reale Accademia per l'Agricoltura, l'Università, la Reale Accademia delle Scienze, il Giardino Botanico e il Museo di Storia naturale. Frescati è stato inoltre il nome di una grande villa inglese, edificata nel 1739 a Blackrock dalla famiglia Hutchinson, anche in questo caso una deliberata corruzione dell'italiano Frascati ${ }^{11}$.

Tornando ai luoghi teatro di battaglie, il caso di Magenta offre un percorso articolato che, concretizzatosi in una duplicazione geografica, introduce però a un processo di lessiconimizzazione che riguarda la replicazione nello spazio linguistico. Scorrendo i paragrafi della voce Magènta dal DI, incontriamo magenta 'color porpora non spettrale, che risulta dalla fusione dei colori delle due estremità dello spettro, il violetto e il rosso; colore cremisi carico' datato 1931 e dal GRADIT più vagamente ma più opportunamente alla seconda metà del sec. XIX; magenta '(in fotografia e in tipografia) colore ottenuto per la composizione additiva dal rosso e dal blu'; magenta 'fuchsina' (1975); rosso magenta (1956); color magenta (1967); lingua magenta, voce medica; bronzo di Magenta 'derivato dal wolfram' (1895). In inglese, segue in nota il DI, Magenta red è documentato dal 1860 e in francese magenta 'nuance de rouge violacé' dal 1862. Anche il DI accredita senz'altro l'ipotesi che il cromonimo alluda al sangue sparso nella bat-

${ }^{10}$ Hotel chiamati Rivoli, per esempio, si segnalano a Roma, Firenze, Sorrento, Cervia (Ravenna), ovviamente Parigi, Monaco di Baviera, Järrenpää e Vantaa in Finlandia, Casablanca, Sharm El Sheik in Egitto, Jounieh in Libano, Gerusalemme, Nuova Delhi, ecc. e si noti il legame con il valore parigino del termine anche nei nomi composti: Villa Rivoli a Nizza, Rivoli Jardin a Helsinki, Rivoli Ramblas a Barcellona.

${ }^{11}$ Dopo lo sviluppo residenziale dell'area denominata Frescati Park, la villa è stata demolita, per favorire la speculazione edilizia, alla fine degli anni 60 del Novecento. 
taglia del 1859, mentre l'Etimologico, ripetendo un'ipotesi accreditata anche da altre fonti lessicografiche, preferisce indicare «prob. con riferimento al colore dei pantaloni dei soldati zuavi dell'esercito francese $»^{12}$.

L'eco mondiale delle battaglie è ben documentata nella storia del nome $\mathrm{Ma}$ genta da Mauro Boscarol e parrebbe legata all'elevato numero di morti e feriti probabilmente dell'intera Seconda guerra d'indipendenza dall'aprile al luglio 1859 che ebbe in Magenta a Solferino i principali teatri di battaglia. Già il 5 agosto 1859 nelle pagine dello «Wisconsin Daily Patriot» un negozio di New York pubblicizzava i Magenta Long Shawls 'scialli lunghi magenta' (e un ristorante di Richmond annunciava che veniva servita una Solferino Soup $)^{13}$. Sappiamo che il colorante sintetico scoperto a Lione proprio nel 1859 e brevettato dalla società Renard Frères \& Franc come fuchsine fu nuovamente brevettato con altro procedimento in Gran Bretagna nel 1860 da Simpson, Maule \& Nicholson come roseine. Questa azienda chiamò il colore della roseina dapprima solferino e poi magenta, a dimostrazione della popolarità dei due toponimi italiani dopo le battaglie del 1859 .

La vittoria, soprattutto da parte francese, ebbe la tradizionale riproduzione a cascata in campo onimico. Non solo tra gli odonimi - il Boulevard de Magenta a Parigi è tra quelli che partono a raggiera da Place de la République - ma anche con le due navi da guerra gemelle francesi costruite nel 1861 con i nomi Magenta e Solferino e con il comune di Dizy-sur-Marne nella Champagne, ribattezzato nel 1881 Dizy-Magenta $^{14}$ (anche negli Stati Uniti figurano 9 siti così denominati, tra cui alcune miniere e corsi d'acqua e almeno tre centri abitati, due in Louisiana e uno in Mississippi) e l'uso antroponimico di Magenta (nome personale femminile in paesi anglofoni e denominazione di un gruppo musicale britannico). Non solo, ma il vincitore di parte francese della battaglia di Magenta del 1859, il capo dell'Esercito Italiano di Napoleone III, Marshal Marie Edme Patrice Maurice de Mac-Mahon, oltre a essersi fregiato del titolo di Maréchal de France dopo quella vittoria, fu da Napoleone III creato $\left(1^{\circ}\right)$ Duca di Magenta. Il titolo nobiliare non è un dettaglio perché Mac-Mahon da allora e sempre più spesso venne chiamato in modo breve Magenta, proseguì la carriera militare e politica, fu governatore generale dell'Algeria e dal 1873 al 1879 presidente della Terza Repubblica francese, dopo Adolphe Tiers e prima di Jules Grévy. Dunque Magenta come predicato nobiliare e infine antroponimo tout-court ebbe una straordinaria popolarità al di là dei valori deonimici del toponimo milanese, di cui si è detto.

${ }^{12}$ Se appare un fatto notevole che il deonimico o transonimo magenta sia documentato già all'indomani della battaglia, sarebbe un fatto linguisticamente del tutto insolito che un cromonimo possa essersi diffuso a motivo della divisa degli zuavi di Napoleone III che parteciparono alla battaglia, tenendo conto che la produzione della fucsina (il cui colore fu appunto detto magenta) ebbe inizio solo pochi giorni prima della battaglia (e ancor meno probabile è che il nome derivi dalle giubbe dei garibaldini che pure combatterono al fianco dei francesi).

${ }^{13}$ Cfr. www.boscarol.com [consultato il 30 aprile 2014].

${ }^{14}$ Dal 1965 Magenta si è separato dal restodel comune. 


\section{Replicazioni nello spazio linguistico}

Ancora un teatro di battaglia è utile per introdurre i percorsi deonimico-transonimici dei toponimi italiani. Cerignola, in Puglia, rappresenta un caso di deonimizzazione di un aspetto particolare della battaglia di cui fu teatro, quello che possiamo definire della 'confusione'. Il valore lessicalizzato di Cerignola, dove gli spagnoli del Gran Capitán, Gonzalo Fernández de Córdoba, sconfissero i francesi del duca di Namours, Louis d'Armagnac nel 1503, in una battaglia breve ma storica, sia per le nuove tattiche e le nuove armi messe in campo, sia perché risultò una tappa fondamentale per il possesso del Regno di Napoli, è sconosciuto all'italiano: il deonimico è esclusivamente iberico. In spagnolo esiste la voce chirinola 'discussione, specie se animata e accalorata', nonché 'schermaglia, lotta' anche 'sciocchezza' o 'festa con giochi, divertimento' e Estar de chirinola vale 'essere pieni di spirito'; pasar la tarde de chirinola 'trascorrere la sera in conversazione'; si tratta di significati piuttosto distanti tra loro, tappe di un degrado semantico: la battaglia armata diventa una scaramuccia, una semplice discussione; poi l'eponimo metonimico non è la battaglia in sé, ma i grandi festeggiamenti che ne seguirono l'esito, con un successivo distanziamento dall'evento in sé, tale che la voce è spesso associata in Spagna alle festività natalizie ${ }^{15}$. Anche nel catalano è penetrata la voce, trasformandola in xerinola. Molti si vantarono d'aver partecipato allo scontro di Cerignola, più di quanti furono realmente presenti; per tale motivo il toponimo è entrato nell'immaginario popolare degli spagnoli da cinque secoli rimanendovi vivissimo.

\subsection{Vulcanonimi e nesonimi}

Nello spazio linguistico, tra processi di lessicalizzazioni e transonimie, possiamo collocare tre nomi di vulcano. Sparsi nel mondo troviamo locali di ristorazione che si rifanno nel nome, e spesso nel logo, al Vesuvio, vulcano nell'area di Napoli; si tratta per la maggior parte di pizzerie che uniscono la tradizione culinaria alla metafora del fuoco; abbondano anche le pasticcerie e i negozi che rivendono pasta e altri prodotti della tradizione alimentare italiana (Vesuvio / Il Vesuvio / Vesuvio Pizza / Vesuvio Cakes / Vesuvio Foods / Chicken Vesuvio). Alcune navi da guerra inglesi e americane sono state denominate Vesuvius, anche qui seguendo la prassi allusiva che accosta la potenza ignivoma dei vulcani al potenziale bellico delle navi. Nel Nord-ovest del Canada si trova la Vesuvius bay (British Columbia) e con lo stesso nome si indica un piccolo centro della Virginia (USA). Una casa discografica scoz-

${ }^{15}$ Per il castigliano si possono riassumere tre significati: fruslería ('bagatela, nadería, pequeñez, nonada'), riña ('disputa, discusión') e fiesta ('juego, alegría, optimismo'). Cfr. www.diclib. com [consultato il 30 aprile 2014]. 
zese attiva negli anni Novanta era ispirata al vulcano (Vesuvius Records) e identico marchionimo identificata anche una casa discografica americana degli anni Sessanta del Novecento (e sempre negli Stati Uniti esistevano le etichette discografiche Volcano e Pompeii); un'antica marca di fiammiferi da cucina fabbricati in Belgio, Vesuvium, era ispirata, con ovvio accostamento simbolico, al vulcano; una birra prodotta dalla compagnia australiana Murray si chiama Vesuvius e nel logo riporta un disegno d'un vulcano in eruzione con la scritta «It goes off» per indicare che "spegne" la sete. Anche l'asteroide $n^{\circ} 13897$ è dedicato al Vesuvio [Randaccio 2015].

L'Etna, la Montagna per antonomasia della Sicilia, denomina - anche nella forma latina Aetna - negli Stati Uniti più di una decina di città e piccoli centri sparsi tra i vari Stati, dalla California alla Georgia, dal Maine all'Ohio. La metafora determinata dalla potenza ignivoma del vulcano ha fatto sì che tra il ' 700 gli inizi del '900 numerose navi da guerra (specie della Marina Inglese) fossero varate con il nome Etna; similmente, anche alcune locomotive delle ferrovie inglesi furono così denominate. La concept-car ideata nel 1984 da Giorgetto Giugiaro per la casa automobilistica britannica Lotus è stata battezzata col nome del vulcano. Anche un modello di telefono cellulare dell'azienda coreana LG è stato dedicato all'Etna ${ }^{16}$.

Infine, Stromboli, che si eleva nell'omonima piccola isola dell'arcipelago siciliano delle Eolie: il nome è associato negli Stati Uniti a un particolare tipo di preparato alimentare chiamato Stromboli; si tratta di una sorta di rollè farcito con vari ingredienti (mozzarella, salumi, pomodoro e verdure). Si dibatte ancora su chi sia l'inventore di questo calzone-sandwich e sul nome che lo individua; due cuochi, entrambi d'origini italiane, si contendono il merito: Nazzareno Romano del "Romano's Restaurant \& Pizzeria" di Essington (Philadelphia) e Mike Aquino del "Mike's Burger Royal" di Spokane (Washington); entrambi sostengono che la scelta del nome fu suggerita dal film di Roberto Rossellini Stromboli (Terra di Dio) del 1950 interpretato da Ingrid Bergman. In seguito, sotto la generica formula Strombolifood si sono via via indicate numerose varianti di questo prodotto. Ricordiamo inoltre che con il nome Stromboli è chiamato, nel film Pinocchio di Walt Disney (1940), il personaggio collodiano di Mangiafoco, con evidente richiamo alla sua natura ignifaga, nonché alla sua "italianità". Sempre per il suo legame con il fuoco e il fumo, alla fine dell'Ottocento fu battezzata Stromboli una grossa loco-

16 Sarà forse, in alcuni casi, una coincidenza, ma con l'acronimo ETNA vengono chiamati l'Electronic Transactions on Numerical Analysis, rivista on-line di matematica della Kent State University (Ohio), l'École des technologies numériques appliquées, con sede a Ivry-sur-Seine (che nutre analoghi interessi legati alla ricerca matematica e ingegneristica), il progetto European Thematic Network on Assistive Information and Communication Technologies, rete tematica europea sulle tecnologie elettroniche e informatiche, la European Tracking Network Association, sistema telematico di informazione tra società assicurative europee per il recupero di auto rubate e l'Extra-Terrestrial Nanomaterials Analysis, progetto universitario (col contributo della "Sapienza" Università di Roma) sui materiali extraterrestri. La Volcano Corporation di San Diego (California), produttrice di strumentazioni e apparecchiature sanitarie, possiede un marchio denominato Etna [Randaccio 2015]. 
motiva delle Great Western Railway, la ferrovia che collegava Inghilterra e Galles (sfruttando l'identica metafora già vista per l'altro vulcanonimo Etna). Infine, il nome del vulcano denomina l'asteroide $\mathrm{n}^{\circ} 26761$.

Tra i nesonimi italiani, Montecristo, piccola isola del Tirreno, è divenuta universalmente nota grazie al romanzo di Alexandre Dumas padre, Il Conte di Montecristo (1846). La reduplicazione del toponimo e la formazione dei deonimici sono connessi alla fama del romanzo, in particolare al valore antonomastico dell'eroe eponimo, ossia quello di 'uomo ricchissimo'. Conseguenza di questa visione metaforica del personaggio e del toponimo inscindibile dal suo titolo nobiliare fu la fondazione di Monte Cristo, villaggio minerario nello Stato di Washington, sorto nel 1890 durante una delle ultime corse all'oro del secolo. Il nome fu scelto, appunto, per richiamare il senso di ricchezza che il romanzo infondeva nella memoria dei pionieri ${ }^{17}$. Montecristo è anche il marchionimo che designa una qualità di sigari cubani; si ipotizza che fabbrica, avviata a Cuba nel 1935 da Alonso Menéndez, abbia scelto il nome perché, durante la lavorazione del tabacco, era abitudine far leggere un libro ad alta voce per intrattenere i torcedores; fra i tanti romanzi letti quello di Montecristo pare fosse così amato dai lavoratori da influenzare il marchio dell'azienda manifatturiera [Randaccio 2013].

Quanto a Capri, nell'arcipelago napoletano, oltre alle località degli Stati Uniti già menzionate, si ricordano tre modelli di automobili che richiamano il nesonimo: la Capri prodotta dalla Lincoln nel 1952, la Capri fabbricata dalla Mercury nel 1970, e la Capri prodotta dalla Ford nel 1969. Nell'immediato Secondo dopoguerra e fino agli anni Sessanta si diffuse la moda di un tipo di pantaloni sportivi da donna, corti al polpaccio e molto attillati creati dalla stilista Sonja de Lennart nel 1948 e chiamati appunto Capri pants. Un tipo di sigarette, prodotte fin dal 1987 dalla Reynolds Tobacco Company, ha analogamente il marchio Capri ${ }^{18}$.

\subsection{Marchionimi}

Come si nota negli esempi dei vulcanonimi e dei nesonimi, l'originario nome geografico s'insinua negli spazi linguistici per diventare tutt'altra cosa, e in più direzioni (transonimia a stella), talvolta perdendo il legame con il referente iniziale, talaltra mantenendo invece un valore semantico comune. Ed è sempre presente in àmbito marchionimico.

${ }^{17}$ Anche le strade e alcuni edifici della cittadina (abbandonata qualche decennio dopo, una volta esaurito il filone aurifero) erano intitolate ai personaggi dumasiani (Dumas street, Mercedes street, Monte Cristo Hotel).

${ }^{18}$ Questa scelta marchionimica, a suo tempo, fece sorgere un contenzioso tra il sindaco di Capri e la multinazionale americana. Il primo cittadino riteneva controproducente l'accostamento della località turistica al tabacco e alle sue dannose conseguenze, rispetto alla vocazione ecologica e salubre dell'isola; cfr. Togliete il nome di Capri da quelle sigarette, "La Repubblica", 18 marzo 1989. 
Tra i marchi aziendali, è notevole il caso del toponimo Montecatini in Val di Cecina (Pisa), che figura, sia pure in forma via via più ridotta, fino alla semplice iniziale, in decine di nomi aziendali, alcuni dei quali molto noti. La storia s'avvia nel 1888 con la ditta Montecatini, che sfruttava le locali miniere di rame; cresciuta notevolmente l'azienda si fuse nel 1966 con la Edison di Milano, nata nel 1895 per l'utilizzo dell'energia elettrica, formando Montedison. Questa si unì a sua volta alla EniChem nel marchio Enimont; tra le società nate nel loro seno (alcune ancora in vita, altre non più), la parola Mont(e) ritorna in Tecnimont (ingegneria), Novamont (bioplastica - il primo elemento del composto è tratto da un altro toponimo, la sede dell'azienda ossia Novara), Montefibre (tecnofibre), Fertimont poi Agrimont (prodotti agricoli), Montedipe (prodotti chimici di base), Montepolimeri (materiali plastici), Alimont (alimentari), Metalmont (metallurgia), Monteshell (petrolifera - con la Shell), Montesud (petrolchimica), Montoil, Simmont, Ausimont (fluoro e tecnoplastiche), Himont (propilene) poi Montell ${ }^{19}$. In altre denominazioni di Montecatini è mantenuta una semplice sillaba, $\mathrm{Mo}$ - in $\mathrm{Mo}$ plefan (Moplen, ossia il propilene che ha rivoluzionato la plastica più cellophane), o una sola lettera di un acronimo (SELM, Società Elettrica Montedison; META, Montedison Terziario Avanzato).

\subsection{Deonimi e sintagmi in altri linguaggi scientifici}

Gli àmbiti nei quali ritroviamo $\mathrm{i}$ toponimi in viaggio sono ancora numerosi.

Geologia: da Milazzo (Messina) deriva il nome di un piano compreso tra quelli chiamati Siciliano e Terraniano; così, mentre il milazzese è un abitante di Milazzo, milazziano ricorda i depositi di calcare arenaceo ricco di fossili marini. Analogamente, il comune alessandrino di Serravalle (Scrivia) ha dato nome a un periodo geologico, il serravalliano, piano del Miocene medio, epoca del Neogene, circa 12-10 milioni di anni fa. Altri piani dei tempi geologici derivano da un comune italiano: tortoniano, messiniano (e zancleano), piacenziano, da Tortona, Messina (già Zanclea) e Piacenza.

Mineralogia: oltre che uno dei capoluoghi di provincia del Lazio, Viterbo è una cittadina in Texas, nella contea di Jefferson, e inoltre un college nel Wisconsin. In Messico esiste una località chiama Santa Rosa de Viterbo che, oltre al toponimo, riprende l'agionimo patronale; in quel luogo fu scoperto e da lì trae nome la viterbite, minerale composto da vari elementi. Da comuni italiani sono denominati anche la iglesiasite, varietà di cerussite; la braccianite, la liparite, la melfite e l'orvietite, tutte rocce eruttive effusive, come pure la ottaianite, dal nome

${ }^{19}$ In Himont la prima sillaba è legata all'azienda americana Hercules che l'ha costituita insieme a Montedison; Montell ha origine dalla fusione con Shell; in seguito divverà Basell con l'acquisizione da parte della Basf, con perdita definitivamente del richiamo Mont-. 
popolare - e ufficiale fino al 1933 - di Ottaviano (Napoli), nonché la sacrofanite, la pantellerite, dall'isola delle Egadi, la sommite e la montesommite derivanti dalla cresta a semicerchio del Vesuvio, l'elbaite dall'isola, la piemontite, la ligurite, la toscanite, la lazialite e la sicilianite da altrettanti coronimi regionali.

Paleontologia: sono noti i resti preistorici del cosiddetto Uomo di Ceprano da un comune del Basso Lazio (o di Argil, perché ritrovato in uno strato di argilla); si tratta del più antico fossile umano rinvenuto sul territorio italiano e l'unico che rappresenti la morfologia (del cranio adulto) dei più antichi abitanti del continente europeo. La nuova specie Homo cepranensis è definita dalla comunità scientifica "il primo abitante d'Europa". Altrettanto significativo è l'Uomo di Pofi, denominazione dei resti rinvenuti, tra il 1959 e il 1961, in una cava pozzolana in località Colle Santa Lucia nel territorio di Pofi (ancora nella provincia di Frosinone).

Economia: Vilfredo Federico Damaso Pareto (1848-1923), di padre italiano esule politico e madre francese, porta il cognome che deriva dal comune di Pareto nell'Alessandrino (Piemonte). Fu ingegnere, sociologo, economista e docente di economia politica all'Università di Losanna e a lui sono legati l'Indice di Pareto (misura delle ineguaglianze nella distribuzione dei redditi), il Principio di Pareto o legge $80 / 20$ (l' $80 \%$ delle ricchezze è in mano al $20 \%$ della popolazione), la $\mathrm{Ma}$ trice di Pareto, l'Efficienza di Pareto, il Diagramma di Pareto, la variabile casuale paretana (in questo caso si tratta di transonimia mediata da un antroponimo).

Medicina: la frazione Valsalva di Castel del Rio nel Bolognese ha originato il cognome portato dall'anatomista e medico imolese Antonio Maria Valsalva (1666-1723). Chirurgo d'avanguardia, considerato il fondatore degli studi della fisiologia dell'orecchio, il suo nome è presente nel linguaggio medico con una decina di denominazioni, che riportiamo in inglese: Teniae of Valsalva, Valsalva's antrum, Valsalva's dysphagia, Valsalva's ligaments, Valsalva's muscle, Valsalva's sinuses, Valsalva's test; la più nota è la manovra di Valsalva ${ }^{20}$.

Botanica: per portare un solo esempio, sono state classificate oltre 60 specie di Sansevieria o Sanseveria, liliacee del genere Agavaceae, originarie del subcontinente indiano o dell'Africa tropicale; traggono il nome da Raimondo di Sangro principe di Sansevero in Puglia (Torremaggiore 1710 - Napoli 1771). Personaggio controverso, inventore e alchimista, letterato e militare, esoterista e massone e cultore di piante esotiche e rare, a lui sono attribuite molte invenzioni. Dal comune foggiano di San Severo ai vegetali dei tropici, il viaggio è breve, grazie alla dedica voluta dal botanico scopritore del genere, Vincenzo Petagna ${ }^{21}$. Lo strumento della mediazione tra toponimo e linguaggio scientifico è qui un predicato nobiliare.

${ }^{20}$ Espirazione forzata a glottide chiusa per compensare le variazioni di pressioni nei cambi di altitudine e nelle immersioni.

${ }^{21}$ Tuttavia non si esclude, oggi, un errore di trascrizione: il naturalista svedese Thumberg ne avrebbe registrato il nome in modo sbagliato, sansevieria anziché sansevierinia (e in tal caso il dedicatario sarebbe stato il duca Pietrantonio Sansaverino). 
Pedagogia: conosciuto in tutto il mondo ${ }^{22}$, il metodo Montessori ha all'origine la pedagogista Maria Montessori, il cui nome di famiglia nasce dalla pluralizzazione di un piccolo toponimo ligure, Montessoro, nel comune di Isola del Cantone (Genova).

Sismologia: Mercallo è in provincia di Varese ed è un altro esempio di toponimo divenuto famoso nel mondo grazie a un cognome, quello di Giuseppe Mercalli (1850-1914), il sismologo che inventò la scala che ne porta il nome.

Si tralasciano le denominazioni di vini, olii, formaggi, salumi, frutti, verdure e altri alimenti individuati dal toponimo di riferimento, nonché le numerosissime altre situazioni in cui scienziati, artisti, personaggi della politica, dello spettacolo, dello sport, delle industrie e del commercio hanno diffuso nel mondo, grazie al loro cognome, i propri toponimi d'origine o quelli dei loro avi: (Leonardo da) Vinci, Caravaggio, (Alessandro) Volta, (Rita Levi) Montalcini, (Camillo) Cavour, (Domenico) Modugno, (famiglia) Prada, (Amedeo e Franco) Modigliani, (Ennio) Morricone, sono un minuscolo campione di toponimi specifici o generici che hanno guadagnato uno spazio linguistico internazionale significativo raggiungendo la popolarità anche se la grande maggioranza dei parlanti nulla o quasi conosce dei referenti originari.

\section{Conclusioni}

Ogni lingua e ogni cultura presenta - in misura e con modalità differenti - un fenomeno analogo a quello descritto per la toponimia italiana. Il fenomeno della trasmigrazione toponimica, ossia della sua replicazione, mostra tre importanti corollari:

a) il primo, che il percorso di un toponimo può riguardare tanto lo spazio geografico e fisico, tanto lo spazio linguistico attraverso i processi di lessicalizzazione, onimizzazione e transonimizzazione, o anche - e i casi non sono rari entrambi gli spazi;

b) il secondo, che la trasmigrazione può avvenire con differenti livelli di apparentamento o allontanamento semantico, e riguardare il lessico comune, i linguaggi scientifici e ogni tipologia di nome proprio;

c) il terzo, che teoricamente qualsiasi toponimo può considerarsi pronto per diventare una voce di lessico o un onimo fungibile e che tali caratteristiche non sono predicibili su base intralinguistica (se non, forse con esclusione di denominazioni troppe complesse, difficile da pronunciare, da memorizzare, e di difficile

${ }^{22}$ Una ricerca in Internet col motore Google, restituisce il nome Montessori in oltre 30 milioni pagine in inglese, 2,5 milioni in russo, 320 mila in cinese, 220 mila in giapponese e quasi 320 mila in islandese [consultato il 15 aprile 2015]. 
articolazione morfologica), bensì su base extralinguistica, ovvero sulla popolarità del nome e sull'utilità del suo riuso in funzione storica, politico-militare, scientifica, pedagogica, orientativa, commerciale. La ragione della trasmigrazione non è data tanto da grandezza fisica, popolosità o altri parametri misurabili tramite indicatori numerici, quanto dall'importanza (generalmente sul piano sincronico) di una data entità geografica, derivante da significatività istituzionale, notorietà politica, religiosa, artistica, architettonica, commerciale e storica ${ }^{23}$.

\section{Bibliografia}

Caffarelli Enzo, 2004, Astrotoponimia del sistema solare. Per un primo approccio descrittivo e metodologico, "Rivista Italiana di Onomastica", 10/2, 415-447.

Caffarelli Enzo, 2013, Il rapporto etimologia-motivazione-uso in toponomastica, [in:] Alberto Manco (ed.), Toponomastica e linguistica nella storia, nella teoria, Napoli, 33-56.

Caffarelli Enzo, 2014, Lessicalizzazioni e transonimie dei toponimi teatri di battaglia, [in:] Giuseppe Brincat (ed.), Onomastica bellica. Da Torino a Malta, Malta, 1-23.

Caffarelli Enzo, 2015a, "Piazza Bologna" a Roma: studio di caso di un panorama onimico. Processi transonimici in macro- e microtoponimi, [in:] Artur Gałkowski, Renata Gliwa (eds.), Mikrotoponimy i makrotoponimy w komunikacji i literaturze, Łódź, 97-112.

Caffarelli Enzo, 2015b, Paparazzo. Il viaggio del cognome italiano più famoso al mondo, Roma, SER.

DEMIM, 2014 = Tiziana Grassi, Enzo Caffarelli et al. (eds.), Dizionario Enciclopedico delle Migrazioni Italiane nel Mondo, Roma.

DI, 1997- = Wolfgang Schweickard, Deonomasticon Italicum. Dizionario storico dei derivati da nomi geografici e da nomi di persona, vol. 3: Derivati da nomi geografici: $M-Q$ (2009), Tübingen.

DTI, 1990 = Giuliano Gasca Queirazza, Carla Marcato, Giovan Battista Pellegrini, Giulia Petracco Sicardi, Alda Rossebastiano (eds.), Dizionario di toponomastica. Storia e significato dei nomi geografici italiani, Torino.

Etimologico, 2010 = Alberto Nocentini (dir.), L'Etimologico. Vocabolario della lingua italiana, Milano.

Gałkowski Artur, Gliwa Renata (eds.), 2014, Mikrotoponimia i makrotoponimia. Problematyka wstępna, Łódź.

Gałkowski Artur, Gliwa Renata (eds.), 2015, Mikrotoponimy i makrotoponimy w komunikacji $i$ literaturze, Łódź.

GRADIT, 1999 = Tullio De Mauro (dir.), Grande dizionario dell'italiano dell'uso, Torino.

La Stella Enzo, 1990, Dalie, dedali e damigiane. Dal nome proprio al nome comune, Dizionario storico di deonomastica, Bologna-Firenze.

Poccetti Paolo, 2014, Microtoponimi e macrotoponimi nell'antichità, [in:] Artur Gałkowski, Renata Gliwa (eds.), Mikrotoponimia i makrotoponimia. Problematyka wstępna, Łódź, 67-88.

Randaccio Roberto, 2013, Quella buon'anima di Montecristo: vicissitudini deonomastiche di un eroe dumasiano, "Rivista Italiana di Onomastica" 19/1, 137-151.

Randaccio Roberto, 2015, Trasmigrazioni toponimiche. Sui nomi di luogo italiani diffusi nel mondo: replicazioni, lessicalizzazioni e transonimie, [in:] Enzo Caffarelli (ed.), Nomi italiani nel mondo. Studi internazionali per i 20 anni della «Rivista Italiana di Onomastica» - Italian Names in the World. International Studies for the 20 Years of «Rivista Italiana di Onomastica», Roma, 183-202.

23 Si veda in proposito anche Poccetti 2014. 


\title{
Enzo Caffarelli
}

\section{Toponymic replications in geographical and linguistic space}

\begin{abstract}
(Summary)
The place names, whatever they refer to, the nature, the history, the settlement size of the referent, often do not stop to designate a single entity, but travel through time and space and that space can have a dual nature: geographical and linguistic. The place name in the geographical space moves through the duplication process and, not frequently, multiple replication, related to the migration of peoples, geomorphological and environmental issues or human settlement, commemorations and tributes, economic and touristic reasons. Instead it moves in linguistic space through two typical and often interconnected processes: lexicalization (from the proper name to the common name) with the subsequent rinomination (from the common name to the proper name) and transonimization (from a proper name to another proper name belonging to another type of onyms). This paper presents examples of Italian macro- and microtoponyms which moved in the geographic space and / or in the linguistic one both in Italy and in Italian language, and, above all, in other languages, nations and continents.
\end{abstract}

Słowa kluczowe: chrematonim, przestrzeń geograficzna, leksykalizacja, przestrzeń językowa, toponim, replikacja toponimiczna, transonimizacja.

Key words: chrematonym, geographical space, lexicalization, linguistic space, toponym, toponymic replication, transonymization. 